\title{
Application of Analytical Electron Tomography to the Study of Pathogenic Protozoa
}

\author{
Kildare Miranda ${ }^{1,2}$, Wendell Girard-Dias ${ }^{1}$ and Wanderley de Souza ${ }^{1,2}$ \\ ${ }^{1}$ Biophysics Institute, Universidade Federal do Rio de Janeiro, Rio de Janeiro, Brazil \\ 2. Instituto nacional de Metrologia, Qualidade e Tecnologia (INMETRO), Rio de Janeiro, Brazil
}

Understanding mechanisms involved in osmoregulation control in protozoan parasites has been a challenge for many research groups. Over the past years, a number of key players in cell signaling in trypanosomatid parasites have been identified. Among these, cyclic AMP (cAMP) has been shown to play a key role in osmoregulation, through a mechanism that involves a cAMP-dependent pathway that leads to the efflux of osmolytes across the parasite surface, and water elimination through a contractile vacuole complex (CVC). In Trypanosoma cruzi, the CVC is formed by a central vacuole surrounded by a collection of interconnected vesicles and tubules that undergo dynamic changes upon osmotic stress. A unique characteristic of this system is the presence of acidic calcium-rich organelles named acidocalcisomes, whose structural organization, chemical properties and physiological activity may vary upon events of osmotic stress. The structure of the osmoregulatory system of Trypnosomes has been described in a few Works [1]. One of the main restrictions for the electron microscopy characterization of this system is that it is highly sensitivity to chemical fixation. This becomes specially critical in trypanosomatids since these cells present a unique osmoregulatory system that seem to congregate a contractile vacuole complex that operates with acidocalcisomes, also been shown to be highly sensitive to chemical fixation [2-4]. To minimize structural changes and ion extraction during sample preparation, cells were submitted to quick freezing and freeze substitution or freeze drying prior to electron microscopy analysis. Sections of freeze-substituted cells or freeze-dried whole cells were submitted to FIB-SEM, serial electron tomography and STEM tomography. For analytical tomography, cells were submitted to plunge freezing and freeze drying. Results showed the detailed organization of the contractile vacuole complex of Trypanosoma cruzi containing a central bladder and a series of tubules that form a spongiome. In addition, a close contact with ion containing organelles named acidocalcisomes was observed. Analytical electron tomography showed an heterogeneous 3D distribution of ions such as phosphate, calcium, magnesium and zinc within the organellar matrix. Electron tomography has tremendously contributed to the understanding of the structural organization of the osmoregulatory system in Trypanosoma cruzi and the use of analytical electron tomography to define the 3D distribution of diffusible ions within the organelles of the parasite demonstrate a clear contribution of this technique to this field of life sciences.

[1] Girard-Dias et al, Histochemistry and Cell Biology 138 (2012),821-831.

[2] Miranda et al, Histochemmistry and Cell Biology 121 (2004), 407-418.

[3] Miranda et al, Protist 155 (2004), 395-405.

[4] Ramos et al., PLoS One 6 (2011), e27276.

[5] This work was supported by grants from MCT-CNPq Universal 480184/2012-7, Coordenação de Aperfeiçoamento do Pessoal de Nivel Superior (CAPES), FAPERJ - Programa Nucleos Emergentes \# E-26/111.185/2011) and Financiadora de Estudos e Projetos-FINEP. 


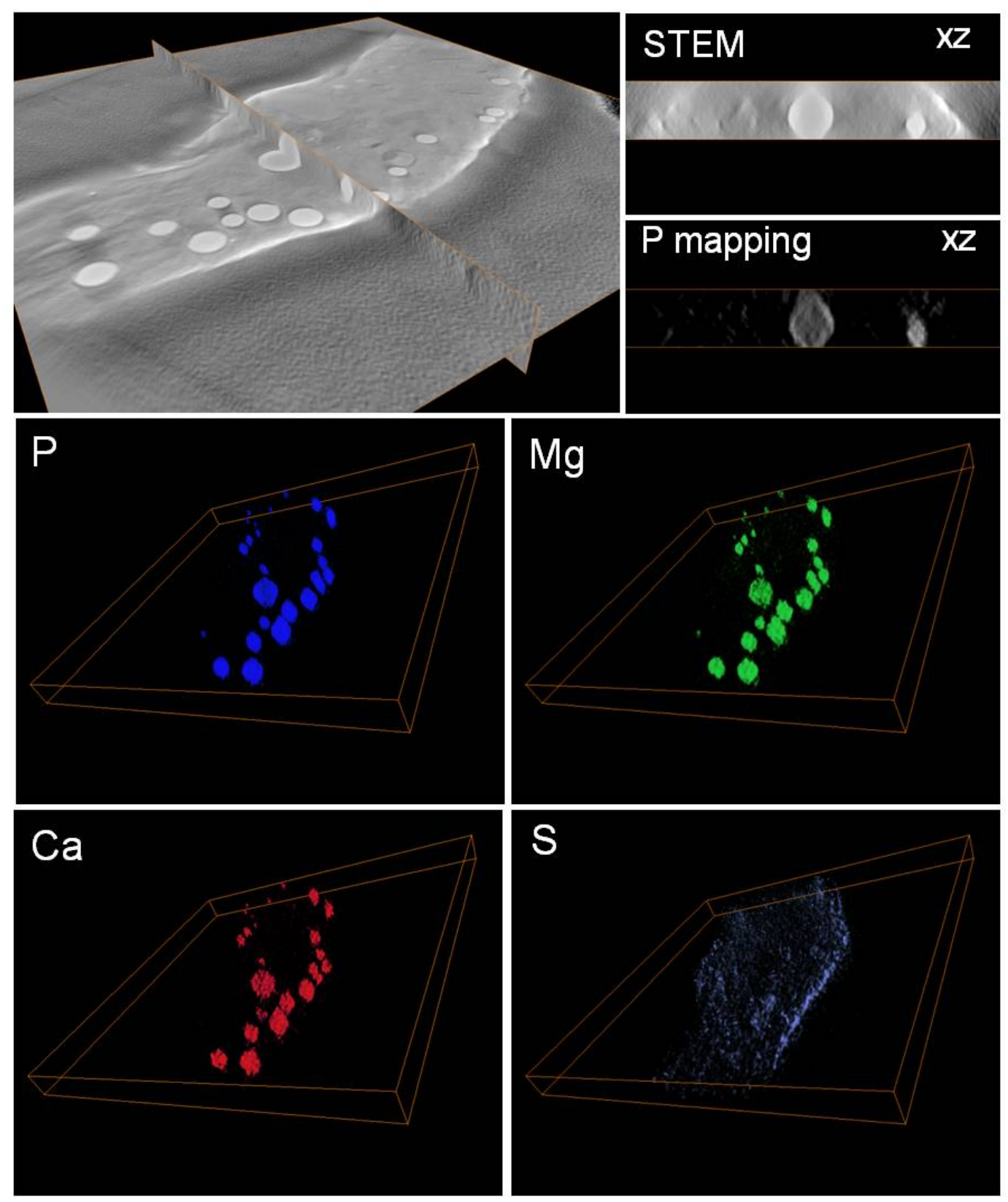

Figure 1. Analytical electron tomography of plunge frozen and freeze substituted Trypanosoma cruzi cells, showing the 3D distribution of phosphorus, magnesium, calcium and sulfur within the parasite body. 